\title{
Improved search for two body muon decay $\mu^{+} \rightarrow e^{+} X_{H}$
}

A. Aguilar-Arevalo, ${ }^{1}$ M. Aoki, ${ }^{2}$ M. Blecher, ${ }^{3}$ D. I. Britton, ${ }^{4}$ D. vom Bruch, ${ }^{5,}$ D. A. Bryman,,${ }^{5,6,{ }^{*}}$ S. Chen, J. Comfort, ${ }^{8}$ S. Cuen-Rochin, ${ }^{6,9}$ L. Doria, ${ }^{6,10}$ P. Gumplinger, ${ }^{6}$ A. Hussein, ${ }^{6,11}$ Y. Igarashi, ${ }^{12}$ S. Ito $\odot,{ }^{2, \uparrow, 8}$ S. Kettell, ${ }^{13}$ L. Kurchaninov, ${ }^{6}$ L. S. Littenberg, ${ }^{13}$ C. Malbrunot, ${ }^{5, \|}$ R. E. Mischke, ${ }^{6}$ T. Numao, ${ }^{6}$ D. Protopopescu, ${ }^{4}$ A. Sher, ${ }^{6}$ T. Sullivan, ${ }^{5, \pi}$ and D. Vavilov,

(The PIENU Collaboration)

\author{
${ }^{1}$ Instituto de Ciencias Nucleares, Universidad Nacional Autónoma de México, CDMX 04510, México \\ ${ }^{2}$ Physics Department, Osaka University, Toyonaka, Osaka 560-0043, Japan \\ ${ }^{3}$ Virginia Tech., Blacksburg, Virginia 24061, USA \\ ${ }^{4}$ SUPA-School of Physics and Astronomy, University of Glasgow, Glasgow G12-8QQ, United Kingdom \\ ${ }^{5}$ Department of Physics and Astronomy, University of British Columbia, \\ Vancouver, British Columbia V6T 1Z1, Canada \\ ${ }^{6}$ TRIUMF, 4004 Wesbrook Mall, Vancouver, British Columbia V6T 2A3, Canada \\ ${ }^{7}$ Department of Engineering Physics, Tsinghua University, Beijing 100084, China \\ ${ }^{8}$ Physics Department, Arizona State University, Tempe, Arizona 85287, USA \\ ${ }^{9}$ Universidad Autónoma de Sinaloa, Culiacán, México \\ ${ }^{10}$ PRISMA ${ }^{+}$Cluster of Excellence and Institut für Kernphysik, Johannes Gutenberg-Universität Mainz, \\ Johann-Joachim-Becher-Weg 45, D 55128 Mainz, Germany \\ ${ }^{11}$ University of Northern British Columbia, Prince George, British Columbia V2N 4Z9, Canada \\ ${ }^{12}$ KEK, 1-1 Oho, Tsukuba-shi, Ibaraki 300-3256, Japan \\ ${ }^{13}$ Brookhaven National Laboratory, Upton, New York 11973-5000, USA
}

(Received 23 February 2020; accepted 16 March 2020; published 31 March 2020)

\begin{abstract}
Charged lepton flavor violating muon decay $\mu^{+} \rightarrow e^{+} X_{H}$, where $X_{H}$ is a massive neutral boson, was sought by searching for extra peaks in the muon decay $\mu^{+} \rightarrow e^{+} \nu \bar{\nu}$ energy spectrum in the $m_{X_{H}}$ mass region 47.8-95.1 MeV $/ c^{2}$. No signal was found and 90\% confidence level upper limits were set on the branching ratio $\Gamma\left(\mu^{+} \rightarrow e^{+} X_{H}\right) / \Gamma\left(\mu^{+} \rightarrow e^{+} \nu \bar{\nu}\right)$ at the level of $10^{-5}$ for this region.
\end{abstract}

DOI: 10.1103/PhysRevD.101.052014

\section{INTRODUCTION}

Observations of neutrino oscillations have established that lepton flavor is not strictly conserved. In the context of

\footnotetext{
* Corresponding author. doug@triumf.ca

Corresponding author. s-ito@okayama-u.ac.jp

*Present address: LPNHE, Sorbonne Université, Université Paris Diderot, CNRS/IN2P3, Paris, France.

${ }^{\S}$ Present address: Faculty of Science, Okayama University, Okayama, 700-8530, Japan.

"Present address: Experimental Physics Department, CERN, Genève 23, CH-1211, Switzerland.

Present address: Department of Physics, University of Victoria, Victoria, BC V8P 5C2, Canada.

Published by the American Physical Society under the terms of the Creative Commons Attribution 4.0 International license. Further distribution of this work must maintain attribution to the author(s) and the published article's title, journal citation, and DOI. Funded by SCOAP ${ }^{3}$.
}

the Standard Model (SM), however, charged lepton flavor violating (CLFV) effects are too small to be observed [1]. Massive or massless weakly interacting neutral bosons $X$ such as axions [2-5] and majorons [6-8] have been suggested to extend the SM including models with dark matter candidates, baryogenesis, and solutions to the strong $C P$ problem. Wilczek suggested such a model [9] which may lead to CLFV where the boson $\mathrm{X}$ can be emitted in flavor changing interactions. Such new bosons have been sought by experiments using kaon [10-15], pion [16,17], and muon decays [18-22].

When decay products from a massive boson $X_{H}$ are not detected due to, for example, a long lifetime, CLFV two body muon decay involving a massive boson $\mu^{+} \rightarrow e^{+} X_{H}$ can be sought by searching for extra peaks in the muon decay $\mu^{+} \rightarrow e^{+} \nu \bar{\nu}$ positron energy spectrum. The mass of the boson $m_{X_{H}}$ can be reconstructed using the equation

$$
m_{X_{H}}=\sqrt{m_{\mu}^{2}+m_{e}^{2}-2 m_{\mu} E_{e}},
$$




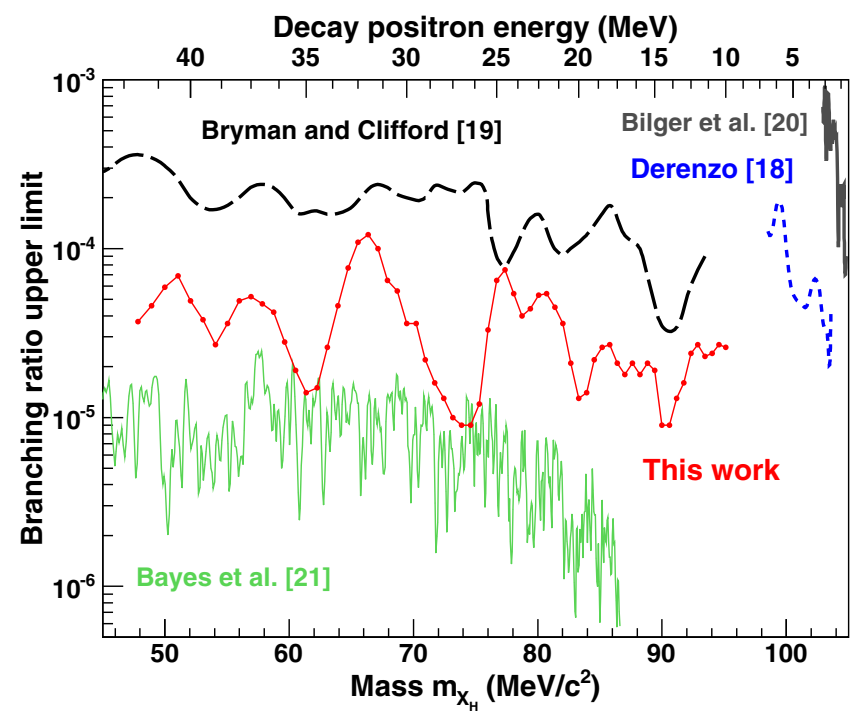

FIG. 1. Summary of the experimental upper limits on the $\mu^{+} \rightarrow e^{+} X_{H}$ branching ratio. The filled red circles with the thin solid red line show the results of this work. The limits represented by the dotted blue line, thick dashed black line, thick solid gray line, and thin solid green line are from Refs. [18-21], respectively.

where $m_{\mu}$ and $m_{e}$ are the masses of the muon and the positron, respectively, and $E_{e}$ is the total energy of the decay positron.

Two-body muon decays $\mu^{+} \rightarrow e^{+} X_{H}$ were searched for by Derenzo [18] using a magnetic spectrometer; experimental limits ${ }^{1}$ on the branching ratio $\Gamma\left(\mu^{+} \rightarrow e^{+} X_{H}\right) / \Gamma\left(\mu^{+} \rightarrow\right.$ $\left.e^{+} \nu \bar{\nu}\right)<2 \times 10^{-4}$ were set in the mass region from 98.1 to $103.5 \mathrm{MeV} / c^{2}$. Exotic muon decays were also sought as a byproduct of the $\pi^{+} \rightarrow e^{+} \nu$ branching ratio measurement [23] by Bryman and Clifford [19] using a $\mathrm{NaI}(\mathrm{T} \ell)$ calorimeter, resulting in upper limits on the branching ratio $\lesssim 3 \times 10^{-4}$ in the mass range from 39.3 to $93.4 \mathrm{MeV} / c^{2}$. Muon decay in the mass region up to the kinetic limit was studied by Bilger et al. [20] using a germanium detector. The most sensitive experiment done so far by Bayes et al. [21] gave limits from $10^{-5}$ to $10^{-6}$ in the mass range from 3.2 to $86.6 \mathrm{MeV} / c^{2}$. Figure 1 shows a summary of the present status of the search for $\mu^{+} \rightarrow e^{+} X_{H}$ decay with upper limits in the mass region from 45 to $105 \mathrm{MeV} / c^{2}$. A massless boson $X_{0}$ was also searched for by Jodidio et al. [22], and the upper limit on the branching ratio was found to be $\Gamma\left(\mu^{+} \rightarrow e^{+} X_{0}\right) / \Gamma\left(\mu^{+} \rightarrow e^{+} \nu \bar{\nu}\right)<2.6 \times 10^{-6}$.

The present work was carried out with data from the PIENU experiment principally designed to measure the branching ratio $\Gamma\left[\pi^{+} \rightarrow e^{+} \nu(\gamma)\right] / \Gamma\left[\pi^{+} \rightarrow \mu^{+} \nu(\gamma)\right]$ using pion decays at rest [24]. A $75 \mathrm{MeV} / c \pi^{+}$beam from the TRIUMF M13 channel [25] was degraded by two thin

\footnotetext{
${ }^{1}$ All limits quoted in this paper are at the $90 \%$ confidence level.
}

plastic scintillator beam counters. Pion tracking was performed by two multiwire proportional chambers and two silicon strip detectors. The pion beam was stopped in an $8 \mathrm{~mm}$ thick plastic scintillator target. Positrons from $\pi^{+} \rightarrow e^{+} \nu$ decays and $\mu^{+} \rightarrow e^{+} \nu \bar{\nu}$ decays following $\pi^{+} \rightarrow$ $\mu^{+} \nu$ decays were measured by two thin plastic scintillators used as telescope counters and a calorimeter consisting of a $48 \mathrm{~cm}($ dia $) \times 48 \mathrm{~cm}$ (length) single crystal $\mathrm{NaI}(\mathrm{T} \ell)$ detector surrounded by pure CsI crystals [26]. A silicon strip detector and a multiwire proportional chamber were used to reconstruct tracks of decay positrons and define the acceptance. The energy resolution of the calorimeter was $2.2 \%$ (FWHM) for $70 \mathrm{MeV}$ positrons. A total of $1.9 \times 10^{8}$ muon decays were used to search for the decay $\mu^{+} \rightarrow$ $e^{+} X_{H}$ with lifetime $\tau_{X}>10^{-9} \mathrm{~s}$. The energy resolution is a factor of 2 improvement, and the statistics are an order of magnitude larger than the previous TRIUMF experiment [19]. The present experiment is also sensitive to a higher mass region than that of Ref. [21].
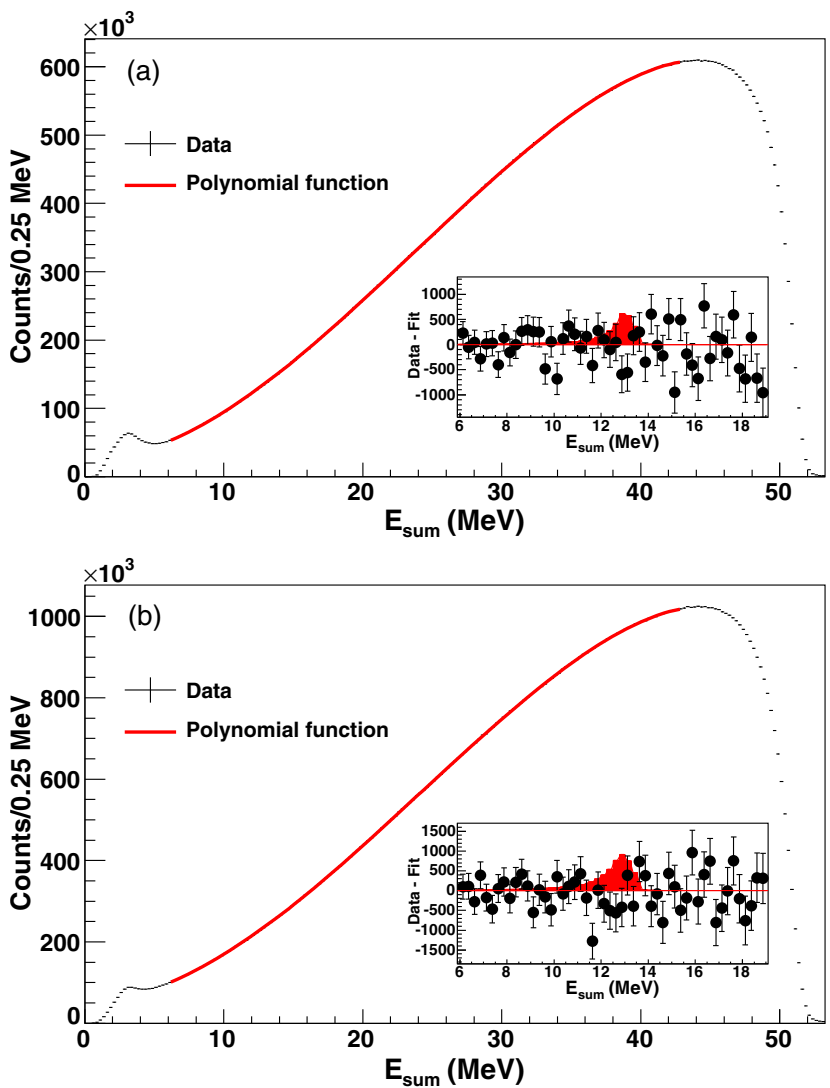

FIG. 2. The muon decay energy spectra from data taken before (a) and after (b) November, 2010 fit to polynomial functions (solid red line). The insert boxes show the residuals in the low energy region with statistical uncertainties (black circles) and a hypothetical signal (from MC) with the branching ratio $5.0 \times 10^{-5}$ with $m_{X_{H}}=90 \mathrm{MeV} / c^{2}$ (red histograms). The bumps at $3 \mathrm{MeV}$ were due to the low energy positrons that hit the telescope counters but did not reach the calorimeter (see text). 


\section{ANALYSIS}

The data in the PIENU experiment were taken in runs occurring from 2009 to 2012. Because the energy calibration system for the CsI crystals was not available before November 2010, the data were divided into two sets, before and after that date. Pions were identified using energy loss information in the beam counters. Any events with extra hits in the beam and telescope counters were rejected. To ensure the events were from muon decay, the late time region $>200 \mathrm{~ns}$ after the pion stop was selected. A solid angle cut of about $15 \%$ was used for the dataset after November 2010. A tighter acceptance cut (corresponding to about $10 \%$ solid angle) was applied to the data taken before November 2010 to minimize electromagnetic shower leakage. Figure 2 shows the muon decay energy spectra for those two datasets where $E_{\text {sum }}$ is the sum of energies observed in the calorimeter, telescope counters, and silicon strip detector including positron annihilation but excluding approximately $1.5 \mathrm{MeV}$ energy loss in the target and inactive materials. The bumps at about $3 \mathrm{MeV}$ in the low energy region of the spectra were due to positrons which hit the telescope counters but did not enter the calorimeter; positron annihilation in the last telescope scintillator resulted in one $0.511 \mathrm{MeV}$ photon depositing energy in the calorimeter.

The two muon decay energy spectra were each fit to smooth sixth order polynomial functions in the energy region $E_{\text {sum }}=6$ to $43 \mathrm{MeV}$ but excluding a region from -1.75 to $+1.25 \mathrm{MeV}$ around a possible signal peak where the search was to be performed. Then, for each $m_{X_{H}}$, the spectra were fit simultaneously to the polynomial functions with fixed fitting parameters obtained in the initial procedure plus a peak signal shape for the decay $\mu^{+} \rightarrow e^{+} X_{H}$. To combine the two datasets, a common branching ratio was used as a free parameter in the fit. The validity of the fit procedure was confirmed using the simulated muon decay energy spectrum and the signal peak with the branching ratio $1.0 \times 10^{-4}$ at several energies. The polynomial function fit without any added signal shape resulted in $\chi^{2} /$ d.o.f. $=1.09$ (d.o.f. $=282$ ). The signal shapes were produced by a Monte Carlo (MC) simulation [27] that reproduced the peak of the decay $\pi^{+} \rightarrow e^{+} \nu$ at $69.8 \mathrm{MeV}$. This procedure was repeated in the range $E_{\text {sum }}=8.5$ to $40.5 \mathrm{MeV}$ (corresponding to the actual decay positron energy $E_{e}=10$ to $42 \mathrm{MeV}$ ) with $0.5 \mathrm{MeV}$ steps.

\section{RESULTS AND CONCLUSION}

No extra peaks due to CLFV muon decay $\mu^{+} \rightarrow e^{+} X_{H}$ with a lifetime $\tau_{X}>10^{-9} \mathrm{~s}$ were observed and upper limits on the branching ratio $\Gamma\left(\mu^{+} \rightarrow e^{+} X_{H}\right) / \Gamma\left(\mu^{+} \rightarrow e^{+} \nu \bar{\nu}\right)$ from $10^{-5}$ to $10^{-4}$ were set for the mass region $m_{X_{H}}=$ 47.8 to $95.1 \mathrm{MeV} / c^{2}$ as shown in Fig. 1. Statistics were the dominant source of uncertainty on the branching ratios. Systematic uncertainties and acceptance effects were approximately canceled by taking the ratio of the fit amplitude of signal events to the number of total muon decays. Improved and new limits in the mass region from 87.0 to $95.1 \mathrm{MeV} / c^{2}$ were set.

\section{ACKNOWLEDGMENTS}

This work was supported by the Natural Sciences and Engineering Research Council of Canada (NSERC, No. SAPPJ-2017-00033), and by the Research Fund for the Doctoral Program of Higher Education of China, by CONACYT doctoral fellowship from Mexico, and by JSPS KAKENHI Grants No. 18540274, No. 21340059, No. 24224006, No. 17H01128, and No. 19K03888 in Japan. We are grateful to Brookhaven National Laboratory for the loan of the crystals, and to the TRIUMF operations, detector, electronics, and DAQ groups for their engineering and technical support. We would also like to thank to R. Bayes and A. Olin for providing the experimental data in Ref. [21].
[1] W. Marciano, T. Mori, and M. Roney, Annu. Rev. Nucl. Part. Sci. 58, 315 (2008).

[2] J. Jaeckel and A. Ringwald, Annu. Rev. Nucl. Part. Sci. 60, 405 (2010).

[3] K. S. Jeong, T. H. Jung, and C. S. Shin, Phys. Rev. D 101, 035009 (2020).

[4] P. Agrawal and K. Howe, J. High Energy Phys. 12 (2018) 029.

[5] D. S. M. Alves and N. Weiner, J. High Energy Phys. 07 (2018) 092.

[6] G. B. Gelmini and M. Roncadelli, Phys. Lett. B 99, 411 (1981).
[7] Y. Chikashige, R. N. Mohapatra, and R. D. Peccei, Phys. Lett. 98B, 265 (1981).

[8] C. S. Aulakh and R. N. Mohapatra, Phys. Lett. B 119, 136 (1982).

[9] F. Wilczek, Phys. Rev. Lett. 49, 1549 (1982); see also A. Davidson and K. C. Wali, Phys. Rev. Lett. 48, 11 (1982).

[10] T. Yamazaki et al., Phys. Rev. Lett. 52, 1089 (1984).

[11] N. J. Baker et al., Phys. Rev. Lett. 59, 2832 (1987).

[12] S. S. Adler et al., Phys. Lett. B 537, 211 (2002).

[13] S. Adler et al., Phys. Rev. D 70, 037102 (2004).

[14] V. V. Anisimovsky et al., Phys. Rev. Lett. 93, 031801 (2004). 
[15] See G. W. S. Hou, J. Phys. Conf. Ser. 800, 012024 (2017) and references therein.

[16] R. Eichler et al., Phys. Lett. B 175, 101 (1986).

[17] C. E. Picciotto et al., Phys. Rev. D 37, 1131 (1988).

[18] S. E. Derenzo, Phys. Rev. 181, 1854 (1969).

[19] D. A. Bryman and E. T. H. Clifford, Phys. Rev. Lett. 57, 2787 (1986).

[20] R. Bilger, K. Föhl, H. Clement, M. Cröni, A. Erhardt, R. Meier, J. Pätzold, and G. J. Wagner, Phys. Lett. B 446, 363 (1999).

[21] R. Bayes et al., Phys. Rev. D 91, 052020 (2015).

[22] A. Jodidio et al., Phys. Rev. D 34, 1967 (1986).
[23] D. A. Bryman, R. Dubois, J. A. Macdonald, T. Numao, B. Olaniyi, A. Olin, J.-M. Poutissou, and M. S. Dixit, Phys. Rev. D 33, 1211 (1986).

[24] A. Aguilar-Arevalo et al., Phys. Rev. Lett. 115, 071801 (2015).

[25] A. Aguilar-Arevalo et al., Nucl. Instrum. Methods Phys. Res., Sect. A 609, 102 (2009).

[26] A. A. Aguilar-Arevalo et al., Nucl. Instrum. Methods Phys. Res., Sect. A 791, 38 (2015).

[27] S. Agostinelli et al. (GEANT4 Collaboration), Nucl. Instrum. Methods Phys. Res., Sect. A 506, 250 (2003); http:// geant4.cern.ch. 